\title{
LAS INVESTIGACIONES DE LA ESCUELA DE CIENCIAS DE LA EDUCACIÓN DE LA UNIVERSIDAD NACIONAL DE ROSARIO
}

\author{
Brenda Caren Bertramo (Universidad Nacional de Rosario** \\ brendabertramo@hotmail.com.ar \\ Agustina Guadalupe Mosso (Universidad Nacional de Rosario)** \\ agustina_mosso@hotmail.com \\ María Isabel Pozzo (Universidad Nacional de Rosario - \\ Consejo de Investigaciones Científicas y Técnicas)*** \\ pozzo@irice-conicet.gov.ar
}

Recibido: 7/08/2012 Aceptado: 10/10/2012

\section{Resumen}

La presente reseña evoca el Foro de Investigaciones de la Escuela de Ciencias de la Educación de la UNR. A partir de la programación del evento, analiza las características de su producción académica en lo que refiere a: composición de los equipos, instituciones acreditadoras y duración total. En los acotados márgenes que impone una reseña, se intenta aportar al relevamiento y diagnóstico de esta importante faceta del quehacer de la Escuela.

\section{Palabras clave}

Investigación - Educación - Universidad.

* Estudiante de la Escuela de Ciencias de la Educación. Facultad de Humanidades y Artes. Univesidad Nacional de Rosario.

** Estudiante de la Escuela de Ciencias de la Educación. Facultad de Humanidades y Artes. Univesidad Nacional de Rosario.

*** Doctora en Ciencias de la Educación por la Universidad Nacional de Rosario. Magister en Formación de Profesores de Español como Lengua Extranjera por la Universitat de Barcelona, España. Profesora y Licenciada en Ciencias de la Educación. Universidad Nacional de Rosario. Investigadora del CONICET, Coordinadora del Área de Estudios Interculturales del Instituto Rosario de Investigaciones en Ciencias de la Educación (IRICECONICET). 
La naturaleza compleja de los fenómenos educativos requiere para su estudio de los aportes de diferentes disciplinas. Es por ello que el campo de investigación en educación presenta una gran diversidad de paradigmas, perspectivas teóricas y metodologías. Esta variedad pudo advertirse a lo largo del "Foro de investigaciones de la Escuela de Ciencias de la Educación", organizado por esta Escuela de la Facultad de Humanidades y Artes de la Universidad Nacional de Rosario y la Asociación de Graduados en Ciencias de la Educación de Rosario (AGCER), durante los días 9 y 10 de mayo de 2012. Junto a la Directora Dra. María Silvia Serra y la Secretaria Técnica Lic. Yanina Fantasía, colaboraron en la organización miembros de AGCER y un grupo de Profesores de la Escuela conformado por: Mg. Natalia Fattore, Dra. María Isabel Pozzo, Dr. José Tranier y Mg. Elisa Welti.

Según se consignó en la circular del evento, sus objetivos fueron:

- $\quad$ Ofrecer un espacio de intercambio entre colegas sobre las investigaciones en curso o recientemente finalizadas.

- Difundir entre los estudiantes y graduados de la carrera las líneas de investigación que se vienen llevando a cabo en el marco de la Escuela.

- Profundizar el camino de la investigación como ámbito de desarrollo profesional del cientista de la educación.

En vistas a cumplimentar estos objetivos, la convocatoria de presentaciones estuvo dirigida a profesores y graduados de la Escuela de Ciencias de la Educación que se encontraran desarrollando investigaciones en el corriente año, o que las hubieran finalizado en 2011, radicadas en centros de investigación reconocidos. Se incluyó asimismo a graduados de la Licenciatura en Ciencias de la Educación que hubieran finalizado sus tesinas en 2011. Al tratarse de investigaciones en curso o recién finalizadas, el evento proporcionó una imagen cabalmente actualizada de la investigación desarrolladas por sus actores. Para promover una amplia participación, el Foro se implementó sin costo alguno, con entrega de certificados y suspensión de clases.

En el transcurso de las dos jornadas se presentaron veintiocho investigaciones en formato de ponencias y posters. Partiendo del programa del Foro y de los resúmenes enviados por los autores, hemos tomado los ejes centrales de la convocatoria para añadir a la evocación del evento una sucinta relatoría.

Las exposiciones fueron agrupadas en mesas por afinidad temática. Igualmente, la diversidad antes mencionada -que se evidencia en la dispersión de las palabras claves - indica la necesidad de adoptar criterios amplios para su sistematización.

Las investigaciones fueron evaluadas y/o subsidiadas por los organismos y en las cantidades que se indican en el gráfico $\mathrm{N}^{\circ} 1$. 


\section{Gráfico №1:}

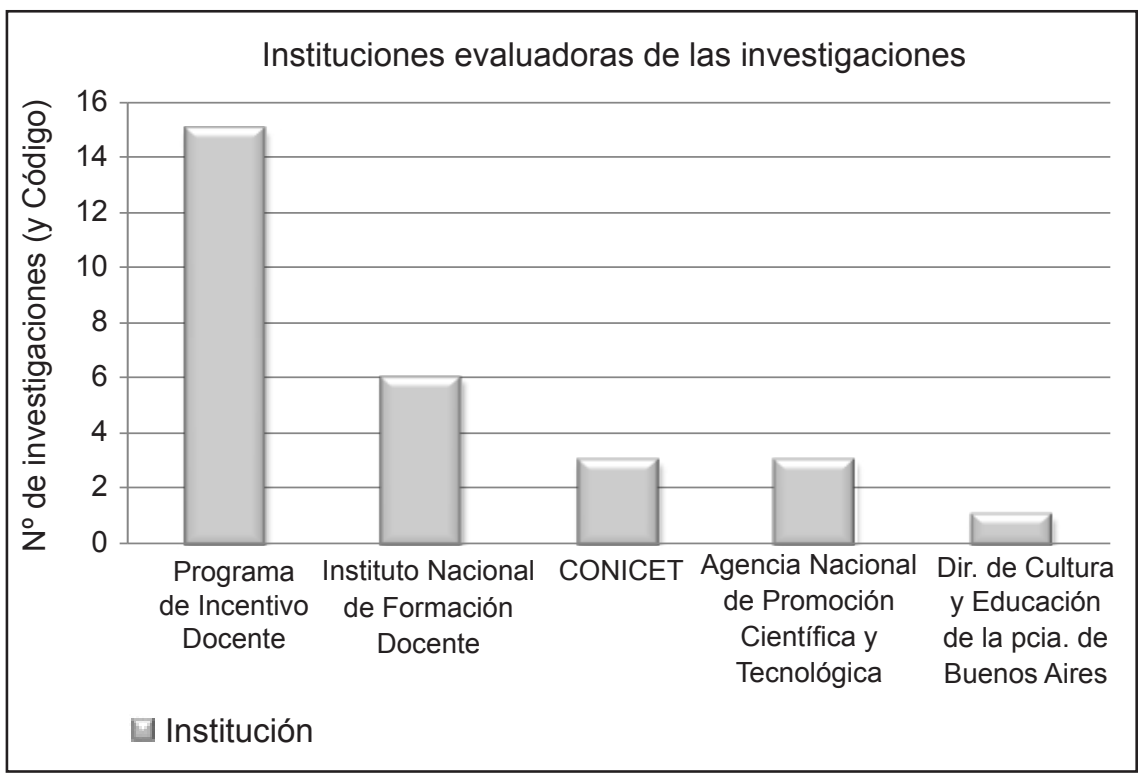

Todas las investigaciones consisten en proyectos grupales, en su mayoría numerosos -entre cinco y diez personas- (gráfico ${ }^{\circ} 2$ ). Es así que los equipos participantes suman más de doscientos miembros, incluyendo los directores, investigadores y estudiantes.

\section{Gráfico $n^{\circ} 2$ :}

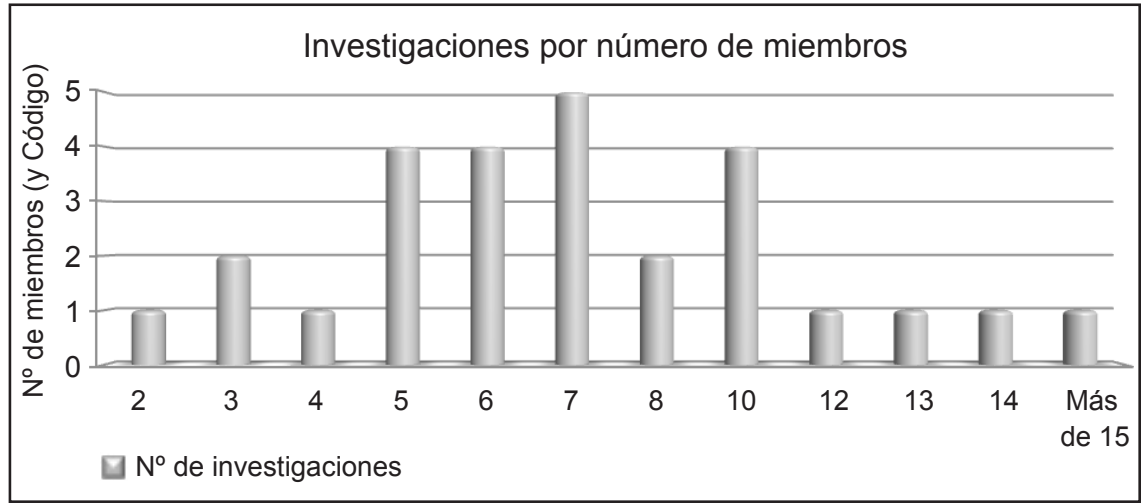


La duración de los proyectos oscila entre 1 y 6 años, según se indica en el gráfico $n^{\circ} 3$. Los proyectos de un año responden a las convocatorias del INFD. El resto se trata de proyectos plurianuales en sus presentaciones iniciales o de continuación.

\section{Gráfico N³:}

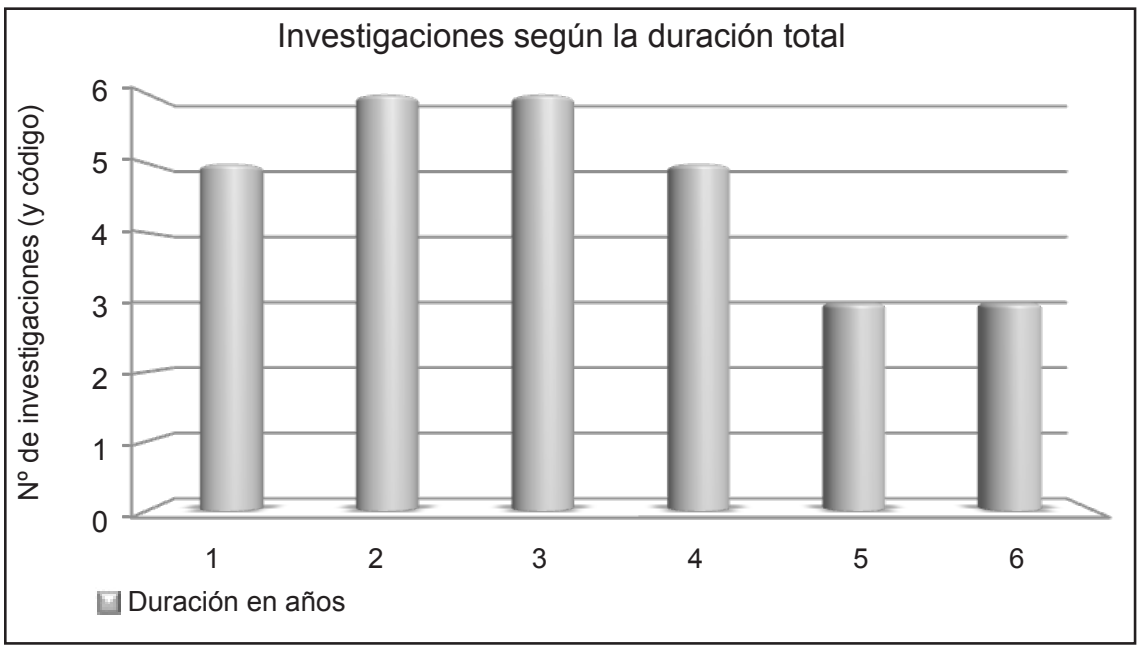

Los participantes -tanto asistentes como expositores- manifestaron un interés sostenido y respaldaron la implementación de este espacio de comunicación. Señalaron además la importancia de reeditar periódicamente este evento, a lo que nosotros agregamos la necesidad de iniciar un registro virtual y/o impreso que lo documente. Como sostiene Claudia Jacinto (2010), la comunicación de los resultados de investigación a los actores del sistema en todos sus niveles debería formar parte de los programas de formación de investigadores en educación (p.303). En el evento que nos ocupa, activar los canales de circulación es indispensable para que la Escuela de Ciencias de la Educación se posicione con perfiles definidos a los fines de la formación y asesoría para la comunidad académica y la sociedad toda. Esto es, qué herramientas teórico-metodológicas puede aportar a las demandas sociales más acuciantes y a las problemáticas vigentes propias del campo. Un trabajo más extenso al respecto requeriría explayarse sobre dimensiones tales como: fortalezas y debilidades de los espacios académicos, contenidos de las investigaciones y metodologías utilizadas, tal como realiza García de Fanelli (2010) a escala nacional. Esta reseña constituye un primer eslabón en tal sentido y anhelamos que otros equipos se sumen a la tarea. 
El Foro incluyó en su programación un homenaje al Prof. Edgardo Ossanna, Profesor Titular de Historia socio-política del sistema educativo argentino del Ciclo de Formación Docente, fallecido en Diciembre de 2010. La segunda jornada finalizó con la presentación de un nuevo número de la Revista de la Escuela de Ciencias de la Educación: №6 - Año 7, 2011. Esta estuvo a cargo de la Directora de la publicación, Mg. Susana Copertari, las integrantes del Comité editorial Mg. Natalia Sgreccia y Lic. María Eugenia Guida y el Prof. Fernando Avendaño, miembro del Comité Académico Interno. Investigaciones y publicaciones constituyen una fórmula indisociable que la Escuela de Ciencias de la Educación supo reunir en el Foro.

\section{Referencias bibliográficas}

- García de Fanelli, A. (2010). ¿Educación o problemas educativos? Reflexiones sobre la construcción del campo educativo. En Wainerman C. y Di Virgilio M. M. (Comps.). El quehacer de la investigación en educación, pp. 293-302. Buenos Aires: Manantial y Universidad de San Andrés.

- Jacinto, C. (2010). La investigación en educación y la toma de decisiones en políticas educativas. En Wainerman C. y Di Virgilio M. M. (Comps.). El quehacer de la investigación en educación, pp. 303-308. Buenos Aires: Manantial y Universidad de San Andrés. 